\title{
Establishing cutoff scores on assessments of surgical skills to determine surgical competence
}

\author{
J. Eric Jelovsek, MD; Mark D. Walters, MD; Abner Korn, MD; Christopher Klingele, MD; \\ Nikki Zite, MD, MPH; Beri Ridgeway, MD; Matthew D. Barber, MD, MHS
}

OBJECTIVE: The aim of this study was to establish minimum cutoff scores on intraoperative assessments of surgical skills to determine surgical competence for vaginal hysterectomy.

STUDY DESIGN: Two surgical rating scales, the Global Rating Scale of Operative Performance and the Vaginal Surgical Skills Index, were used to evaluate trainees while performing vaginal hysterectomy. Cutoff scores were determined using the Modified Angoff method.

RESULTS: Two hundred twelve evaluations were analyzed on 76 surgeries performed by 27 trainees. Trainees were considered minimally competent to perform vaginal hysterectomy if total absolute scores (95\% confidence interval) on Global Rating Scale $=18$ (16.5-20.3) and Vaginal Surgical Skills Index $=32$ (27.7-35.5). On average, trainees met new cutoffs after performing 21 and 27 vaginal hysterectomies, respectively. With the new cutoffs applied to the same cohort of fourth-year obstetrics and gynecology trainees, all residents achieved competency in performing vaginal hysterectomy by the end of their gynecology rotations.

CONCLUSION: Standard-setting methods using cutoff scores may be used to establish competence in vaginal surgery.

Key words: assessment, competence, global assessment of operative performance, vaginal hysterectomy, vaginal surgery, vaginal surgical skills index

Cite this article as: Jelovsek JE, Walters MD, Korn A, et al. Establishing cutoff scores on assessments of surgical skills to determine surgical competence. Am J Obstet Gynecol 2010;203:81.e1-6.

$\mathrm{T}$ o carry out their charge of protecting the public, licensing and certifying organizations must develop and administer assessment instruments that distinguish between trainees with ade-

From the Obstetrics, Gynecology, and Women's Health Institute, Cleveland Clinic (Drs Jelovsek, Walters, Zite, Ridgeway, and Barber), Cleveland, OH; the Department of Obstetrics and Gynecology (Dr Korn), University of California, San Francisco, School of Medicine, San Francisco, CA; and the Department of Obstetrics and Gynecology (Dr Klingele), Mayo Clinic, Rochester, MN.

Presented at the 35th Annual Meeting of the Society of Gynecologic Surgeons, New Orleans, LA, March 30-April 1, 2009.

Received Aug. 13, 2009; revised Oct. 5, 2009; accepted Jan. 24, 2010.

Reprints: J. Eric Jelovsek, MD, Cleveland Clinic, Department of Obstetrics and Gynecology, Building A81, 9500 Euclid Ave., Cleveland, OH 44195. jelovsj@ccf.org.

This study was supported by the Multi-Center Education Research Award, Society of Gynecologic Surgeons.

0002-9378/\$36.00

(C) 2010 Mosby, Inc. All rights reserved. doi: 10.1016/j.ajog.2010.01.073 quate and inadequate levels of knowledge and skill. ${ }^{1}$ Unfortunately, surgical skills in obstetrics and gynecology are not directly assessed by these organizations. Although the American Board of Obstetrics and Gynecology requires a trainee to pass a written and oral examination that allows them to demonstrate they "know how" to perform surgical skills, it assumes that trainees can appropriately "perform" these skills. There is no requirement for these organizations to directly observe performance; rather, direct observation during surgery occurs by supervising surgeons during residency or fellowship. Trainees are often deemed competent to perform procedures based on nonvalidated, subjective or objective global assessments and case $\operatorname{logs} .^{2}$ However, these forms of assessment do not provide direct evidence that an individual competently performs a skill, because case logs lack content validity and most forms of global assessment have poor reliability and unknown validity. ${ }^{2}$

Therefore, the challenge continues for surgeon educators to establish more robust methods of determining surgical competency that truly reflect safe and ef- fective surgical care. Competency-based learning begins with setting standards. There are several methods of standard setting and they can be broadly categorized as relative (norm referencing) or absolute (criterion referencing). Norm referencing describes an individual's performance relative to his or her position within a group. For example, a resident is judged by comparison to the scores achieved by his or her resident colleagues on the same test. Although this is the most common method of referencing, it aims to rank trainees and allows trainees to be compared with one another. However, it cannot provide a clear assessment of the trainee's abilities, because there will always be a fixed number who fail. Moreover, norm referencing encourages competition, not cooperation, and it is somewhat unstable, as it will shift according to the performance of the norm group. ${ }^{3}$ Criterion referencing provides a clear definition of what the trainee should be able to do and it provides a given standard to indicate a competent level of performance. Criterion referencing is also more responsive to the subject matter being taught and allows the teacher and learner to clearly pinpoint capabilities. 
A common procedure for obstetrics and gynecology trainees to learn and become competent performing is hysterectomy. One in 5 hysterectomies in the United States is performed using the vaginal approach. ${ }^{4}$ Vaginal surgery is considered the approach of choice for most patients requiring hysterectomy, because morbidity appears to be lower with the vaginal approach than with any other method. ${ }^{5,6}$ Therefore, it is imperative for obstetrics and gynecology trainees to be competent performing vaginal hysterectomy. Our group recently determined the validity and reliability of 2 assessment scales that resident and fellow training programs may use to objectively assess intraoperative vaginal surgical skills: the Global Rating Scale (GRS) of operative performance, developed by Reznick et $\mathrm{al}^{7}{ }^{7}$ and the Vaginal Surgical Skills Index (VSSI), specifically designed for the evaluation of vaginal surgical skills. ${ }^{8}$ The primary aim of this study was to use formal standard-setting techniques to establish credible and defensible minimum cutoff scores on these intraoperative assessments of surgical skills to determine competence performing vaginal hysterectomy.

\section{Materials and Methods}

Institutional review board exemption was obtained, as this project involved the use of educational tests and did not affect the clinical course of patients. This is a supplemental study to our original study in which we determined the reliability and validity of 2 scales that can be used to assess trainees performing vaginal surgery: the new VSSI and the GRS of operative performance. To summarize our original study, the GRS was developed by Reznick et $\mathrm{al}^{7}$ and consists of a 7-item global rating scale that allows supervising surgeons to directly rate important but generic skills during surgical procedures. The GRS is currently the only recommended assessment instrument listed in the Accreditation Council for Graduate Medical Education's Assessment Toolbox for assessing surgical skills. This instrument has good internal consistency (Cronbach's alpha $=0.95$ ) and acceptable intrarater reliability (in- traclass correlation coefficient [ICC], 0.64 ) but low interrater reliability (ICC, 0.31) for assessing a trainee's surgical skills while performing vaginal surgery. The VSSI consists of 13 surgical principles. The VSSI also has good internal consistency (Cronbach's alpha $=0.96$ ), acceptable intrarater reliability (ICC, 0.82 ), and better interrater reliability (ICC, 0.53) than the GRS during the assessment of trainees performing live vaginal surgery. Both instruments appear to be valid assessment scales for vaginal surgery, because they demonstrate convergent validity (how closely a new scale is related to other measures of the same construct to which it should be related) and discriminate validity (ability to distinguish between training levels).

The GRS (range, 0-35) and VSSI (range, 0-52) were used to evaluate postgraduate trainees while performing vaginal hysterectomy in obstetrics and gynecology from 2 academic medical centers. A $10-\mathrm{cm}$ visual analog scale (VAS) indicating a trainee's overall level of surgical performance was also completed. A higher score on all scales indicates better performance. Vaginal hysterectomies were performed by obstetrics and gynecology residents and $\mathrm{Fe}$ male Pelvic Medicine and Reconstructive Surgery fellows (postgraduate years [PGY], 1-7) between May 2007-June 2008 and were observed live and video recorded to validate a new intraoperative surgical scale for vaginal surgery, the VSSI. Trainees performed a vaginal hysterectomy while the procedure was videotaped in a blinded, standardized fashion. An expert surgeon scored the trainee using all 3 assessment scales immediately after the procedure and again 4 weeks after the procedure using the videotape. A second blinded surgeon at a third participating institution evaluated all the videotapes using the same scales.

Because the aim of this study was to establish credible and defensible minimum cutoff scores on these 2 valid and reliable intraoperative assessments of surgical skills, methods of credible standard setting and procedures for establishing defensible absolute passing scores on performance examinations in health profession education were applied to the assessment scales. ${ }^{1,9}$ Content expert surgeons included 7 gynecologic surgeons representing the East, South, Midwest, and West Coast of the United States from 3 different academic medical centers, including: 4 from the Cleveland Clinic, 1 from Mayo Clinic, 1 who recently located to the Cleveland area from the University of Tennessee at Knoxville, and 1 surgeon from the University of California, San Francisco. Two of 7 experts were women, all were board-certified obstetricians/gynecologists, and 4 of the 7 finished an American Board of Obstetrics and Gynecology/American Urological Association-approved fellowship in Female Pelvic Medicine and Reconstructive Surgery. All experts were familiar with assessment tools, curricula, and trainees. Historically, most standardsetting studies have demonstrated that judges, absent all performance data, tend to set unrealistically high passing scores, which will fail an unreasonably high proportion of trainees. To avoid this, it is recommended that experts are "calibrated" for the standard-setting methods to have a realistic expectation of actual trainee performance by first-hand observation of the actual scale scores for trainees and in-depth discussion of differences between competence and expertise. ${ }^{9,10}$ This was performed for this study.

Most absolute standard-setting methods are based around the concept of a borderline trainee's performance. The concept of a borderline trainee originated from Angoff and assumes that a borderline trainee is one who has an exactly 50:50 probability of passing or failing the assessment. ${ }^{10}$ Therefore, a minimum cutoff score is 1 that separates those who are competent and those who are not. For this study, cutoff scores were determined using a single method, the Modified Angoff method, and confirmed using scores derived from 2 additional standard-setting methods, the Contrasting Groups method ${ }^{11,12}$ and Hofstee method. ${ }^{10,13,14}$ Each of these standard-setting methods is described later.

In this study, the Modified Angoff method began as the experts discussed the characteristics and gave examples of 
TABLE

Minimum acceptable cutoff scores on 3 intraoperative assessments of vaginal surgical skills

\begin{tabular}{llll} 
Method of standard setting & VSSI & GRS & VAS \\
\hline Modified Angoff & $32(62 \%)$ & $18(50 \%)$ & $51(51 \%)$ \\
& $95 \% \mathrm{Cl}, 27.7-35.5$ & $95 \% \mathrm{Cl}, 16.5-20.3$ & $95 \% \mathrm{Cl}, 39.6-62.4$ \\
\hline Contrasting groups $^{\mathrm{a}}<$ or $\geq 20$ vaginal hysterectomies & $32(62 \%)$ & $21(60 \%)$ & $47(47 \%)$ \\
\hline Hofstee $^{\mathrm{a}}$ & $34(65 \%)$ & $20(57 \%)$ & $49(49 \%)$
\end{tabular}

Data are presented as mean absolute score (\% of total score).

Cl, confidence interval; GRS, Global Rating Scale; VAS, visual analog scale; VSSI, Vaginal Surgical Skills Index.

a Unable to calculate confidence intervals.

Jelovsek. Establishing cutoff scores on assessments of surgical skills. Am J Obstet Gynecol 2010.

a borderline trainee performing vaginal hysterectomy. Experts came to an agreement on these borderline characteristics, and each expert determined the score a minimally competent trainee should get on each item on the 3 assessment scales. A discussion among the experts then ensued based on each judge's rating. Experts were given the opportunity to change their scores if desired. Scores were averaged for each item and summed to determine the minimum passing score for this method. To determine the level of agreement of the new cutoff scores between experts, the interobserver reliability was assessed using the ICC.

The minimum cutoff scores obtained by the Modified Angoff approach were then compared with scores obtained using 2 additional methods of standard setting. Furthermore, because volume of surgical cases is often clinically used as a surrogate of determining competence, we elected to use volume alone as a possible separating characteristic in 1 of the standard-setting methods. In our experience, the volume for vaginal hysterectomy is approximately 20 cases. This clinical impression is supported by a recent investigation looking at the development of proficient operating times using a newly implemented robotic approach for hysterectomy with lymph node dissection by gynecologic oncologists. ${ }^{15}$ Using the Contrasting Groups method, we divided trainees into competent ( $\geq 20$ vaginal hysterectomies) and noncompetent $(<20$ vaginal hysterectomies). The distributions of scores were plotted from the 3 intraoperative scales, and the Contrasting Groups method was applied to each scale. The passing score was set at the intersection of the distribution of the 2 groups assuming false-negative and false-positive errors were of equal weight. The cutoff scores are the ones that best discriminate between the 2 groups. The Contrasting Groups method has been previously applied to assessing laparoscopy skills and is the basis for the widely used cutoff values in the Fundamentals of Laparoscopy Skills curriculum required of all graduating General Surgery residents. ${ }^{16}$

The Hofstee method ${ }^{13,14}$ of standard setting is sometimes referred to as the "relative-absolute compromise method," because it combines features of both relative and absolute standard setting. ${ }^{1}$ For this method, the actual distributions of scores, including the mean, standard deviations, and quartiles, were presented in itemized and graphical form and discussed with the experts. After experts came to an agreement on the characteristics of a borderline trainee, 4 Hofstee questions were presented and discussed, with each judge understanding the implications of each question: What is the lowest acceptable percentage of trainees to fail the procedure (minimum fail rate); What is the highest acceptable percentage of trainees to fail the procedure (maximum fail rate); What is the lowest acceptable percent-correct score for each assessment scale that allows a borderline trainee to pass the procedure (minimum passing score); and What is the highest acceptable percent-correct score for each assessment scale that allows a borderline trainee to pass the procedure (maximum passing score)? The mean percentage across all experts was calculated and each point was plotted as a line on a cumu- lative frequency distribution of scores for each scale. The midpoint of the minimum and maximum failure rates and pass scores represented the overall competency cutoff score for this method for all experts. All analyses were performed using JMP 7.0 (SAS Institute, Cary, NC).

\section{Results}

The 212 evaluations were analyzed on 76 surgeries performed by 27 trainees, and all evaluations were assumed to be independent of one another for purposes of this analysis. The Table summarizes cutoff scores on the 3 assessment instruments using all 3 standard-setting methods. Based on the Modified Angoff method, trainees should be considered minimally competent to perform vaginal hysterectomy if total absolute scores on VSSI $=32$ (95\% CI, 27.7-35.5), GRS = $18(95 \%$ CI, 16.5-20.3), or VAS $=51$ (95\% CI, 39.6-62.4). The level of agreement of the new cutoff scores between experts (the interobserver reliability) was high, with the ICC $=0.81$.

To verify these findings, the Contrasting Groups approach to standard setting was used on data from all 3 assessment scales. Figure 1 demonstrates how the cutoff score was determined for the VSSI using this approach. Overall, trainees who performed $\geq 20$ vaginal hysterectomies achieved absolute VSSI scores ( $\%$ of total score $)=32(62 \%)$, GRS $=21$ $(60 \%)$, and VAS $=47(47 \%)$, confirming the values reached using the Modified Angoff method.

Using the third standard-setting method, the Hofstee method, experts were provided with data from the study period, including range of scores, means, 


\section{FIGURE 1 \\ Contrasting Groups standard- setting method using surgical volume to determine a minimum acceptable cutoff score on the VSSI}

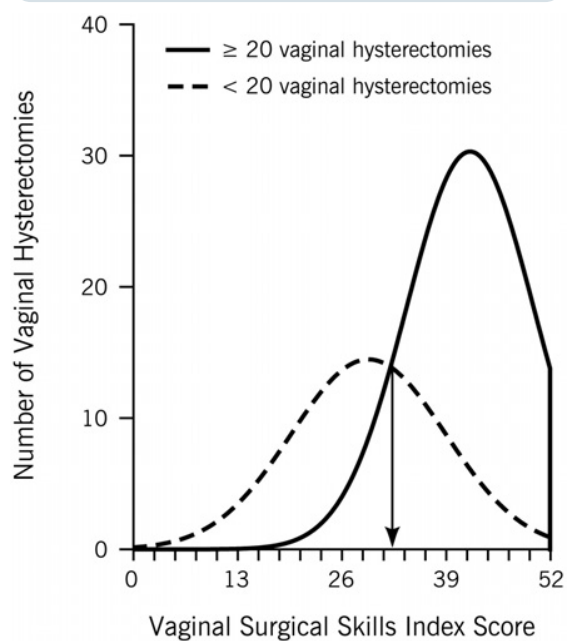

In the Contrasting Groups method of standard setting, trainees were divided into competent ( $\geq 20$ vaginal hysterectomies [solid line]) and noncompetent $(<20$ vaginal hysterectomies [dashed line]). The distributions of VSSI scores were plotted and the passing score was set at the intersection of the distribution of the 2 groups, assuming false-negative and false-positive errors were of equal weight, as demonstrated by the solid arrow.

VSSI, Vaginal Surgical Skills Index.

Jelovsek. Establishing cutoff scores on assessments of surgical skills. Am J Obstet Gynecol 2010.

measures of error, and quantiles in tables and graphically as distribution. The cutoff point was set, as defined by the Hofstee method, where a diagonal line of the calculated answers intersected the cumulative frequency distribution plot (Figure 2). Based on the Hofstee method, trainees should be considered minimally competent to perform vaginal hysterectomy if total absolute scores on VSSI = $34(65 \%)$, GRS $=20(57 \%)$, or VAS $=49$ (49\%). These values also confirmed the values reached using the Modified Angoff method.

Because surgical volume had a moderate correlation with assessment scores, GRS $(r=0.58)$, VSSI $(r=0.66)$, and VAS $(r=0.66)$ scores (all $P<.001)$, and because the Modified Angoff method was our standard-setting method of choice, we wanted to determine the actual number of vaginal hysterectomies that would be required to reach the new cutoff scores on the 2 intraoperative assessment scales. On average, trainees met the new competency cutoffs on the GRS and VSSI after performing 21 and 27 vaginal hysterectomies, respectively. Furthermore, if the new cutoff scores were applied to the same cohort of fourth-year obstetrics and gynecology trainees, 5 of the 9 would have been considered noncompetent in performing vaginal hysterectomy at some point during the rotation, but all residents achieved competency by the end of their fourth-year gynecology rotations.

\section{Comment}

This study has demonstrated the feasibility and use of formal standard-setting methods to establish credible and defensible minimum cutoff scores on scales for assessing surgical skills. These findings are timely, because there has been a recent shift away from norm-referenced assessment of training in the health professions to a criterion-referenced system, where the concern is to graduate physicians who have demonstrated, using valid and reliable assessment methods, the required competence to perform certain skills. ${ }^{17,18}$ To say that a graduating surgeon is competent is no longer enough. The trainee must be competent to do $a$, or $a$ and $b$, where $a$ and $b$ are specific aspects of the surgeon's work. Although there are areas of knowledge, skills, and attitudes common to all doctors regardless of specialty, clearly there are areas unique to the area in which we specialize. $^{17}$

Determining competent performance in vaginal surgery first requires setting some standard. Formal standard setting is used to ensure that the trainee who passes a cutoff score has mastered the core skills, or competencies, that are assessed by the examination, in this case the examination being his or her performance rated on the intraoperative scales. In the standard-setting process of educational testing, terms such as credible and defensible, rather than valid, are
FIGURE 2

Minimum competency cutoff scores for the VSSI during vaginal hysterectomy using the Hofstee method

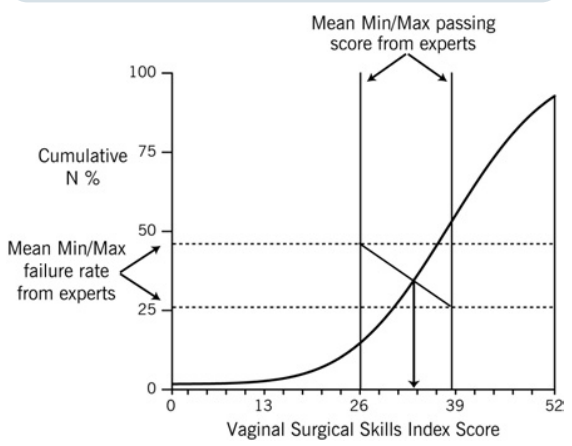

A cumulative frequency distribution of VSSI scores is plotted. The 2 solid vertical lines represent the mean lowest/highest acceptable passing score, as determined by all experts, and the 2 horizontal dotted lines represent the lowest/highest acceptable failure rates. The midpoint of the intersection of the minimum and maximum failure rates and the lowest and highest pass scores for the group of experts (arrow) represents the overall competency cutoff score using this method for the VSSI scale by the Hofstee method.

VSSI, Vaginal Surgical Skills Index.

Jelovsek. Establishing cutoff scores on assessments of surgical skills. Am J Obstet Gynecol 2010.

used when discussing the determination of pass-fail points, because a performance standard, or pass-fail point, is arbitrary and subject to educational or social judgments. Thus, they can never be tested to establish the "correctness" of the passing score. As a result, rather than speaking of validity, it makes more sense to focus on technical considerations and the accumulated evidence to support the use of a particular standard for a particular purpose. ${ }^{1}$ In other words, it makes more sense to write about the evidence in support of the "credibility" of the standard, rather than to validate it, because validation is not possible in standard setting. $^{1}$

The Angoff approach to standard setting is the most widely used method in medicine to determine a cutoff point on a scoring scale that separates the noncompetent from the competent trainee. ${ }^{10}$ It is also easily adapted to performance examinations. 
The method, like all standard-setting methods, is a judgmental approach in which a group of experts make estimates about how borderline candidates would perform on items in an examination or assessment. ${ }^{19}$ Because content experts make judgments about every prompt or checklist item, it is easy to defend the resulting passing scores. In addition, the Angoff method was the first of the absolute methods and thus has the longest history of successful use, even in high-stakes testing situations such as the United States Medical Licensing Examination. ${ }^{3}$ The Modified Angoff method allows the experts to have actual performance data from a scale as an additional source of information to make a decision. Most standard setting in medicine uses the modified version of the Angoff method, because it is a reasonable, practical, and defensible approach to standard setting. ${ }^{20}$ For all these reasons, we elected to use this approach as our primary method of determining a minimum cutoff value on the assessment scales.

Vaginal hysterectomy is a competence required of all graduates of obstetrics and gynecology training programs. ${ }^{17}$ This study has established credible and defensible minimum cutoff scores (VSSI $=32$, GRS $=18$, and VAS $=51$ ) that institutions may use to determine whether a trainee is minimally competent to perform a vaginal hysterectomy. For this project, we should emphasize that the aim of determining these scores is to establish a pass-fail point or, in other words, determine the minimum level of operative performance necessary to provide an acceptable level of surgical service. The proposed cutpoints can be used to distinguish the trainee who is competent to perform vaginal hysterectomy from one who is not. Presumably, a higher set of cutoff scores could be developed to distinguish the "merely competent" from the experienced or expert surgeon.

These data provide objective evidence that training institutions may use to reassure health care regulators and the public that trainees have reached a minimum level of competence during their training. Reassurance is provided through by knowing that establishing surgical competency was determined us- ing systematic approaches of standard setting rather than traditional, unsystematic approaches. At our institution, the impact has been to reassure trainees that they have or have not reached a point in their training where they should be able to perform a vaginal hysterectomy in unsupervised practice. This allows them to focus during the remainder of their training on continuing to improve their technique with this procedure or to use their time to focus on procedures in which they feel less proficient. It is also provides valuable feedback for trainees as they progress in training, because benchmarks in surgical procedures are often perceived as a zone of uncertainty for trainees who encounter marginal surgical volume. This is one reason why we recommend using either the GRS or the VSSI rather than the VAS, which does not provide specific targeted areas of improvement, making it less useful for feedback.

Although we recognize that surgical volume alone does not necessarily equate with surgical competency, on average, trainees who perform $21-27$ vaginal hysterectomies should reach the minimum cutoff scores described in this study. Because residents' opportunities to perform vaginal hysterectomy may be limited by the service they rotate on, it would be important to know how other methods of deliberate repetitive practice, such as simulation or mental imagery, might also improve these scores. These alternative methods may be useful for programs with limited surgical volume.

Before institutions or accrediting bodies accept these values, it is important to consider several important characteristics of standard-setting methods. Different standard-setting methods will produce different passing scores; there is no gold standard. ${ }^{9}$ The Modified Angoff method asks participants to determine the borderline trainee's performance on specific items within the different scales. This is often referred to as a test-centered, or item-centered, method, as it requires experts to make judgments about the expected performance of borderline competent examinees on selected items. ${ }^{21}$ We believed that these itemcentered methods were most useful for setting a pass-fail score on individual assessment, such as performing a vaginal hysterectomy. On the contrary, this may not be the best method to determine who passes or fails a full rotation in gynecology. In this case, it may be better to use the Hofstee method, because this method is considered a compromise method, as it combines some elements of a relative standard and other elements of an absolute standard. ${ }^{13}$ For example, this approach has been used to set a standard for the overall surgery clerkship grade for medical students. ${ }^{22}$ However, it is unclear if high-stakes decision makers should determine surgical competence from a single surgical assessment vs multiple assessments, where a trainee can score low in 1 assessment but compensate for this poor score by scoring higher in another assessment of surgical skill. For example, if a program director has a graduating resident who has some competent scores and some noncompetent scores for a procedure, our suggestion would be to use a weighted average of the most recent set of scores and use additional methods of assessment to make a final decision.

Misapplication of these cutoff scores could result in unacceptably high failure rates, leading training programs or credentialing boards to deal with the issue of multiple failures and the need for retesting. The decision to use such cutoff scores as part of board certification remains unanswered and largely depends on whether the minimum performance scores are acceptable to the stakeholders (eg, public, board examiners, surgeons, and credentialing bodies). Furthermore, decisions on remediation for those trainees who fail also need to be addressed in future studies before widespread implementation.

Institutions should consider whether these cutoff scores would be acceptable by practicing gynecologists at their own institution. The key to defending acceptable standards lies in the choice of credible judges and in the use of a systematic approach to collecting their judgments. ${ }^{9}$ We carefully chose experts in both general gynecology and subspecialty gynecology from locations around the country. The experts in this study were 
"calibrated" to have a realistic expectation of actual trainee performance. Experts almost always expect too much of novice trainees, and if these experts do not have a clear understanding of what constitutes marginal surgical skills compared with an experienced surgeon's skills, cutoff scores may become too strict, resulting in higher failure rates on the assessments. The standards chosen for this evaluation may be different from gynecologists practicing in community settings, making application of these scores in this setting unclear. An additional limitation to all standard-setting methods is that they require some aspect of judgment. In an effort to deal with this, most experts agree that bcause there is no "true" standard, many standard setters with appropriate qualifications should be involved. Therefore, before institutions make formal decisions on competency we recommend repeating the assessment several times using different assessors. Furthermore, these cutoff scores only apply to vaginal hysterectomy. Whether these scores will apply more globally to other vaginal procedures is currently unknown. Future studies will determine whether program directors or board examiners will be able to use such cutoff values to report that a trainee is competent to perform 1 procedure and not another.

Overall, standard-setting methods can be applied to assessments of surgical skills to establish minimum cutoff scores to determine surgical competence. Rigorous methods should be used to determine the most appropriate standard-set- ting method, and experts should be carefully chosen. As new assessment instruments become widely adopted, institutions should develop and improve methods to determine competence to reassure the public that we are graduating safe and effective gynecologic surgeons.

\section{REFERENCES}

1. Norcini JJ, Shea JA. The credibility and comparability of standards. App Meas Educ 1997;10:39-59.

2. Reznick RK. Teaching and testing technical skills. Am J Surg 1993;165:358-61.

3. Nungester RJ, Dillon GF, Swanson DB, Orr NA, Powell RD. Standard-setting plans for the NBME comprehensive part I and part II examinations. Acad Med 1991;66:429-33.

4. Wu JM, Wechter ME, Geller EJ, Nguyen TV, Visco AG. Hysterectomy rates in the United States, 2003. Obstet Gynecol 2007;110: 1091-5.

5. American College of Obstetricans and Gynecologists. Appropriate use of laparoscopically assisted vaginal hysterectomy. ACOG committee on gynecologic practice bulletin no. 311 . Obstet Gynecol 2005;105:929-30.

6. Johnson N, Barlow D, Lethaby A, Tavender E, Curr E, Garry R. Surgical approach to hysterectomy for benign gynaecological disease. Cochrane Database of Syst Rev 2008;4: CD003677.

7. Reznick R, Regehr G, MacRae H, Martin J, McCulloch W. Testing technical skill via an innovative "bench station" examination. Am J Surg 1997;173:226-30.

8. Chen C, Korn A, Klingele C, et al. Objective assessment of vaginal surgical skills. Am J Obstet Gynecol 2010. In Press.

9. Downing SM, Tekian A, Yudkowsky R. Procedures for establishing defensible absolute passing scores on performance examinations in health professions education. Teach Learn Med 2006;18:50-7.

10. Angoff WH. Scales, norms, and equivalent scores. In: Thorndike RL, ed. Educational mea- surement, 2nd ed. Washington, DC: American Council on Education; 1971:508-600.

11. Burrows PJ, Bingham L, Brailovsky CA. A modified contrasting groups method used for setting the passmark in a small scale standardised patient examination. Adv Health Sci Educ Theory Pract 1999;4:145-54.

12. Clauser BE, Clyman SG. A contrastinggroups approach to standard setting for performance assessments of clinical skills. Acad Med 1994;69(suppl 10):S42-4.

13. Hofstee WKB. The case for compromise in educational selection and grading. In: Anderson SB, Helmick JS, eds. On educational testing. San Francisco: Jossey-Bass; 1983:107-27.

14. De Gruijter DN. Compromise models for establishing examination standards. J Educ Meas 1985;22:263-9.

15. Seamon LG, Cohn DE, Richardson DL, et al. Robotic hysterectomy and pelvic-aortic lymphadenectomy for endometrial cancer. Obstet Gynecol 2008;112:1207-13.

16. Fraser SA, Klassen DR, Feldman LS, Ghitulescu GA, Stanbridge D, Fried GM. Evaluating laparoscopic skills: setting the pass/fail score for the MISTELS system. Surg Endosc 2003; 17:964-7.

17. Dunn WR, Hamilton DD, Harden RM. Techniques of identifying competencies needed of doctors. Med Teach 1985;7:15-25.

18. Bienstock JL, Robinson RD, Bennett TL, et al, eds. Educational objectives: a core curriculum in obstetrics and gynecology, 9th ed. New York: Professional Publishing Group Ltd.; 2009. 19. Cisek GJ. Standard setting guidelines. Educational Measurement Issues and Practice 1996;15:12-21.

20. Kaufman DM, Mann KV, Muijtjens AM, van der Vleuten CP. A comparison of standard-setting procedures for an OSCE in undergraduate medical education. Acad Med 2000;75: 267-71.

21. Cusimano MD. Standard setting in medical education. Acad Med 1996; 71(suppl 10):S11220.

22. Schindler N, Corcoran J, DaRosa D. Description and impact of using a standard-setting method for determining pass/fail scores in a surgery clerkship. Am J Surg 2007;193:252-7. 